\title{
BILATERAL MIDDLE CEREBRAL ARTERY STROKE TREATED WITH A DOUBLE THROMBECTOMY. REPORT OF TWO CASES.
}

Ramos, C. ${ }^{1}$; Aguirre, C. ${ }^{1}$; Trillo, S. ${ }^{1}$; Villacieros Álvarez, J.1; Martínez-Vicente, L. ${ }^{1}$; Caniego Monreal, J.L 2; Bárcena Ruiz, E.2; Ximénez-Carrillo, A. ${ }^{1}$; Vivancos, J. ${ }^{1}$

Stroke Unit. Department of Neurology. La Princesa Research Institute. La Princesa University Hospital, Spain.

2 Department of Radiology. La Princesa University Hospital, Spain.

\section{BACKGROUND:}

Bilateral middle cerebral artery (MCA) occlusion in patients with acute ischemic stroke (AIS) is an extremely rare and catastrophic entity, whose treatment has not been established. We are reporting two cases of acute bilateral MCA occlusion treated with a double mechanical thrombectomy (MT) at our center.

\section{CASOS CLINICOS:}

\section{CASE 1}

A 68 year-old female with history of atrial fibrillation (AF) treated with antiplatelets presented with impaired level of consciousness, aphasia and right-sided hemiparesis (NIHSS:12) with unknown onset time.

Baseline CT showed a bilateral hyperdense MCA sign and loss of gray-white matter differentiation in the left lenticular nucleus (Fig.1). CT perfusion (CTP) was performed to identify the extension of tissue at risk for infarction (Fig. 2) and to evaluate the size of the infarct core. CT Angiography (CTA) showed a total occlusion of the proximal M1 segment of the left MCA with another occlusion in the proximal M2 segment of the right MCA (Fig.3).

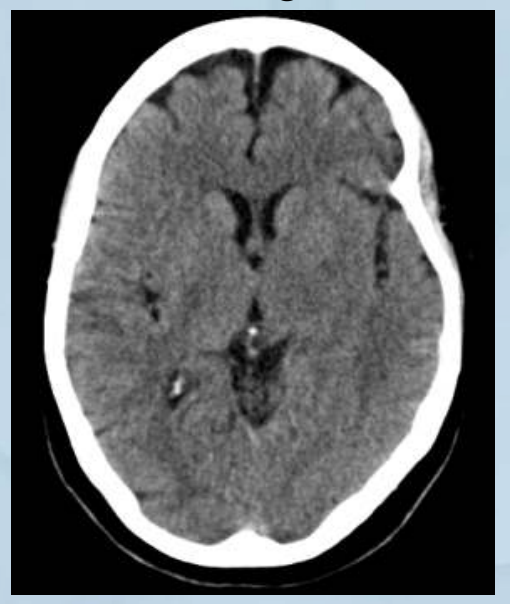

Fig.1: Baseline CT

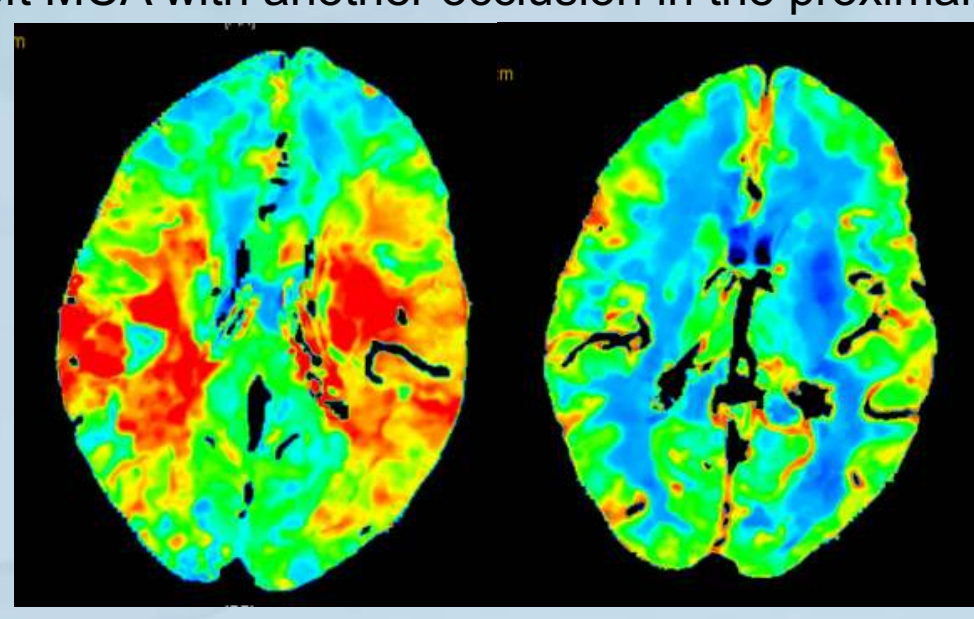

Fig. 2: CTP

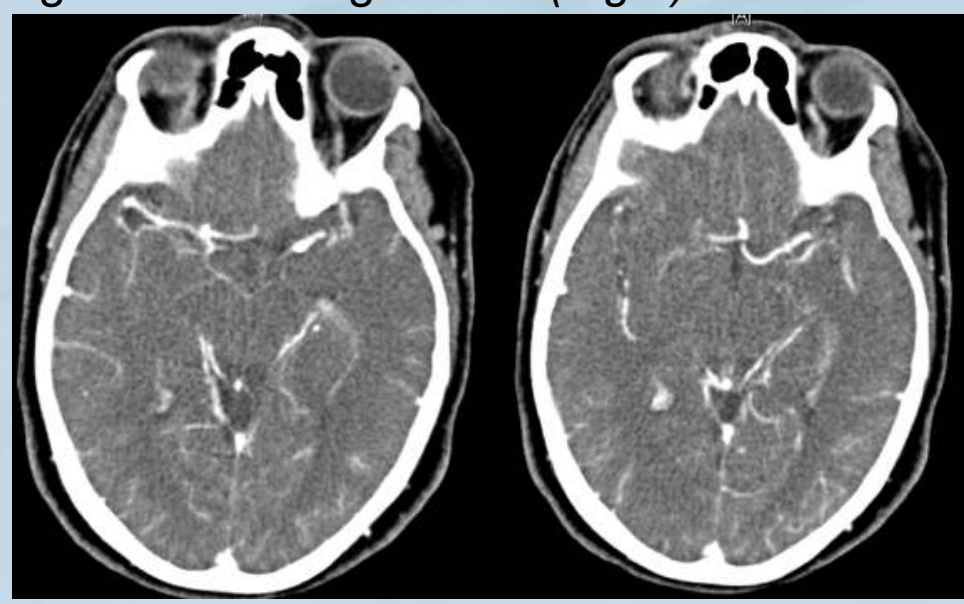

Fig. 3: CTA

Endovascular treatment was initiated eighteen hours from last time seen well, achieving early recanalization of the left MCA (Fig. 4). However, right MCA remained occluded despite multiple stent-triever passes (Fig. 5). Follow-up CT after 24 hours (Fig. 6) demonstrated a bilateral cerebral infarction with hemorrhagic transformation HI2 (1). Anticoagulant therapy was started two weeks after stroke onset. Three months after the stroke the patient was dependent in daily life activities (modified Ranking Scale:4).

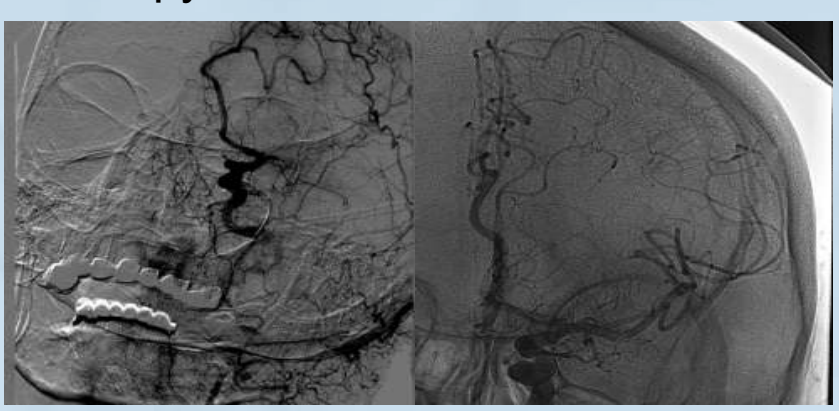

Fig. 4: Left MCA

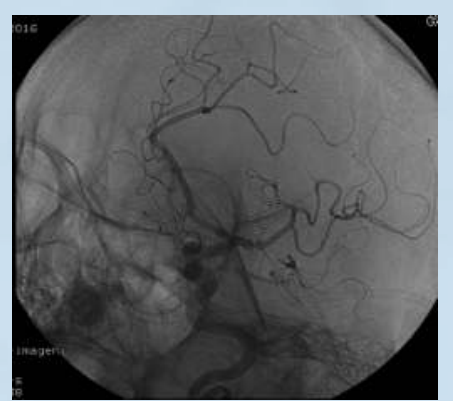

Fig. 5 : Right $M C A$

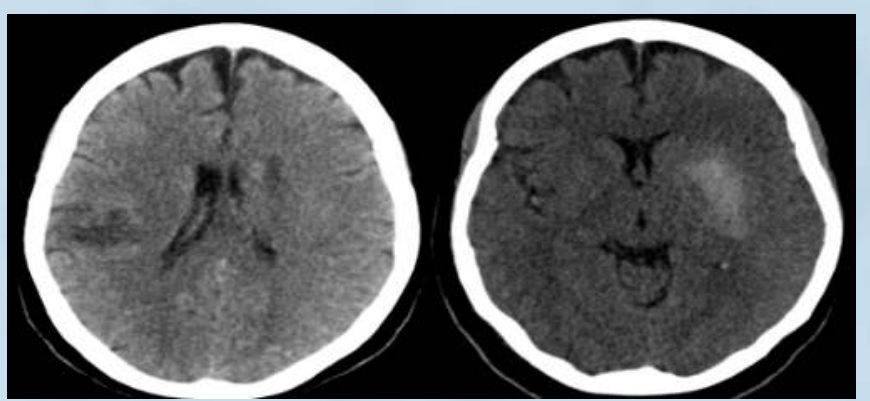

Fig. 6: Follow-up CT

\section{CASE 2}

Case2: An 82 year-old female with history of hypertension, hypercholesterolemia and diabetes under antiplatelet therapy was admitted to the emergency room with acute onset of aphasia and left-sided hemiparesis (NIHSS:16).

Baseline CT (Fig.7) was normal (ASPECTS:10) and intravenous thrombolysis was started without neurological improvement. CTA (Fig. 9) showed a double artery occlusion (left M1 segment and right carotid T) partly compensated by leptomeningeal collateral circulation (LMC) with an extensive area of brain hypoperfusion and a small infarct core assessed by CTP (Fig.8).

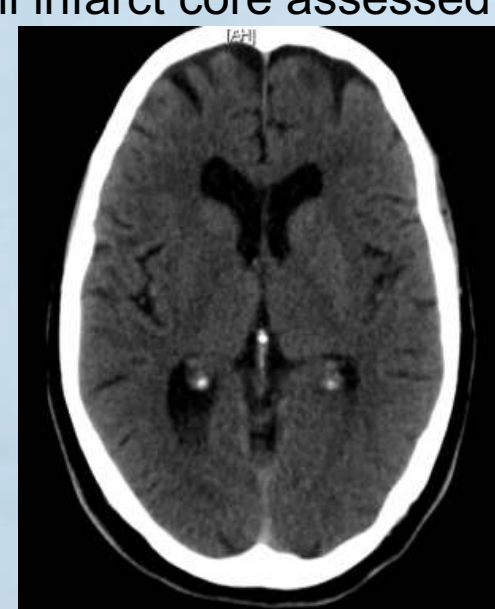

Fig. 7 : Baseline CT

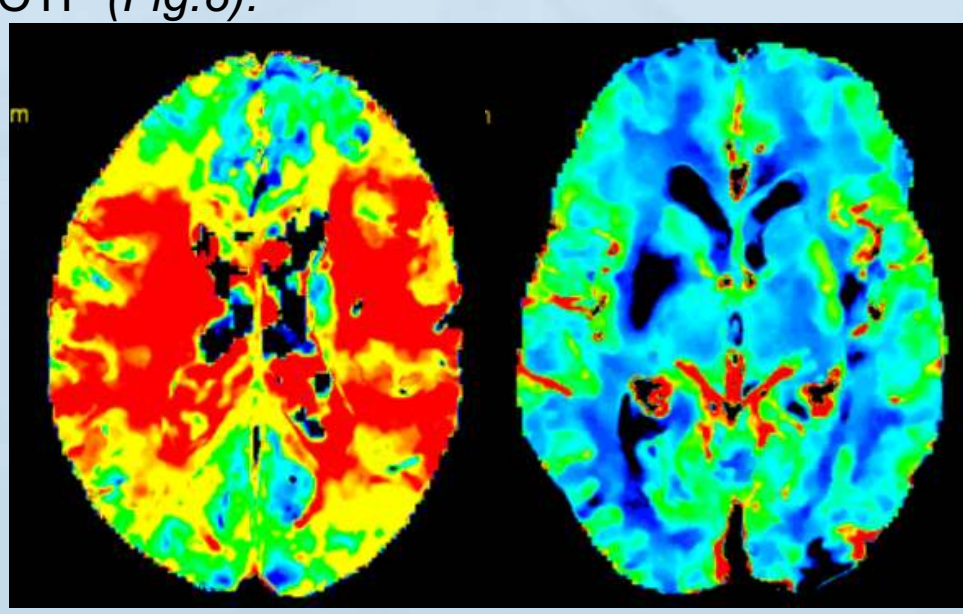

Fig. 8: CTP

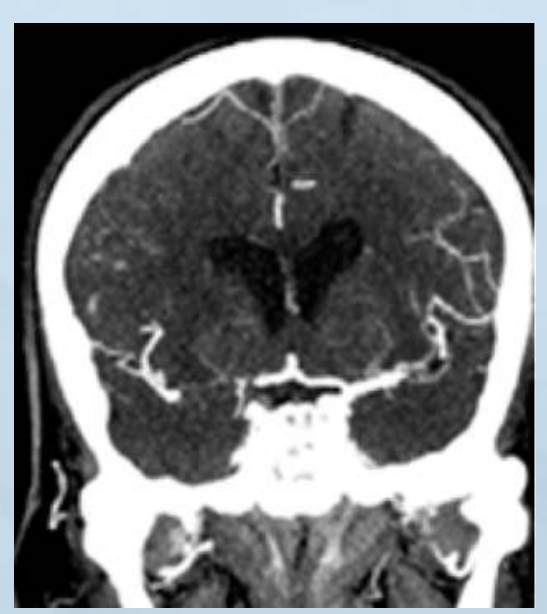

Fig. 9: CTA

Both arteries were recanalized ( $\mathrm{TICl}$ III) within six hours of stroke onset (Fig. 10). Nevertheless, the patient did not show neurological recovery and she was admitted to the intensive care unit. Brain CT after 24 hours (Fig. 11) demonstrated an extensive bilateral infarction and a transthoracic echocardiogram (TTE) showed a severely dilated left atrium (LA) without other abnormalities. The patient's condition deteriorated due to a severe pneumonia and she passed away ten days from stroke onset.
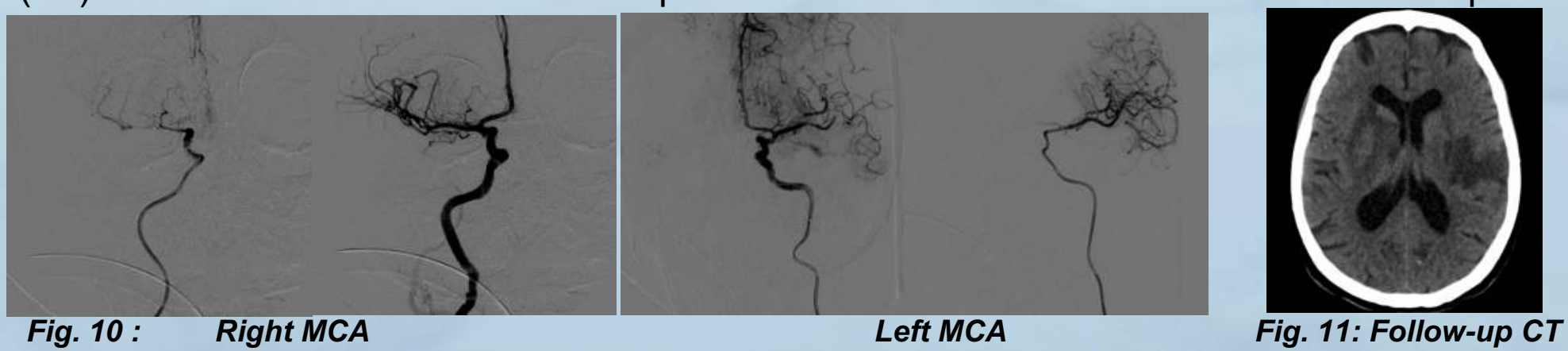

\section{CONCLUSIONS:}

Stroke due to acute bilateral MCA occlusion is a rare entity difficult to diagnose with unknown prognostic factors and high morbimortality despite successful treatment. However, despite the high mortality rate, successful treatment was possible on one of our patients that otherwise probably would have died. We have not found similar cases of AIS due to bilateral MCA occlusion in literature treated with a double MT at the same time. More cases are needed to identify specific prognostic factors and to assure the best possible treatment of these patients. 